\title{
Comparison of Efficacy of Adjuvant MEC (Methotrexate, Epirubicin and Cisplatin) and GC (Gemcitabine and Cisplatin) in Advanced Upper Tract Urothelial Carcinoma
}

\author{
SHENG-CHUN HUNG ${ }^{1}$, SHIAN-SHIANG WANG ${ }^{1,2,3}$, CHUN-KUANG YANG $^{1}$, JIAN-RI LI $^{1}$, \\ CHEN-LI CHENG $^{1}$, YEN-CHUAN OU ${ }^{1}$, HAO-CHUNG HO ${ }^{1}$, KUN-YUAN CHIU $^{1}$ and CHUAN-SHU CHEN ${ }^{1}$ \\ ${ }^{1}$ Division of Urology, Department of Surgery, Taichung Veterans General Hospital, Taichung, Taiwan, R.O.C.; \\ ${ }^{2}$ School of Medicine, Chung Shan Medical University, Taichung, Taiwan, R.O.C.; \\ ${ }^{3}$ Department of Applied Chemistry, National Chi Nan University, Puli, Taiwan, R.O.C.
}

\begin{abstract}
Aim: To evaluate the efficacy of methotrexate, epirubicin and cisplatin (MEC) or gemcitabine and cisplatin $(G C)$ as adjuvant chemotherapy in advanced upper tract urothelium carcinoma (UTUC). Patients and Methods: From 2002 January to 2008 December, a total of 70 patients with advanced UTUC received radical nephroureterctomy at our Institute with MEC and GC as adjuvant chemotherapy. Diseasefree survival (DFS), cancer-specific survival (CSS) and overall survival (OS) among the two groups were evaluated. Results: The MEC $(n=30)$ and $G C$ group $(n=40)$ were compared and showed no significant differences in DFS $(p=0.859)$, CSS $(p=0.722)$ and $O S(p=0.691)$. Positive lymph nodes, preoperative creatinine $>1.5$, old age, a high ECOG state and low BMI are all the independent risk factors of poor prognosis in advanced UTUC. Conclusion: In patients with UTUC, MEC has a non-inferior efficacy to GC in consideration of cancer recurrence, cancer-specific survival and overall survival.
\end{abstract}

The worldwide incidence rate of urothelial carcinoma is 8.9 per 100,000 person-years for men and 2.2 per 100,000 person-years for women (1). Bladder tumors have been estimated at about $90-95 \%$ (urothelial carcinomas) and is the most common malignancy in the urinary tract (2). Upper tract urothelial carcinomas (UTUC) are relatively rare and account for only $5-10 \%$ of UCs, but are common in Taiwan

This article is freely accessible online.

Correspondence to: Chuan-Shu Chen, Division of Urology, Department of Surgery, Taichung Veterans General Hospital, Taichung, Taiwan, R.O.C. Postal code 40705, No. 1650, Sec. 4, Taiwan Boulevard, Taichung, Taiwan, R.O.C. Tel: +886975358995 , Fax: +886 4-23592525-5121, e-mail: r2060d@yahoo.com.tw

Key Words: Upper tract urothelial carcinoma, adjuvant chemotherapy.
(3). Its behavior appears multi-focal and in $17 \%$ of cases, concurrent bladder cancer is present (4). Furthermore, an estimated $22-47 \%$ of patients with UTUC may suffer from bladder recurrence (5) and $2-6 \%$ of patients suffer from contralateral recurrence (6).

A radical nephroureterectomy and bladder cuff excision is standard treatment for UTUC, while offering the benefits of preventing tumor spreading and an entire urinary tract resection (7). However, after definite and en bloc surgical removal of the kidney, it still appears to be a poor prognosis for advanced disease, with a $<50 \%$ 5-year cancer-specific survival rate in pT2/pT3 tumors and $<10 \%$ in pT4 patients $(8,9)$.

For adjuvant chemotherapy, current practice is mainly focused on evidence related to muscle-invasive bladder cancer. Despite the similar morphology between upper and lower tract bladder tumors (10), the phenotype and genotype still showed differences between the two groups (11). Cisplatin-based chemotherapy used in bladder cancer may not always be suitable for patients with UTUC due to potential nephrotoxicity. Current analysis also suggests that data generated from bladder urothelial carcinoma cannot always be extrapolated to patients with UTUC $(12,13)$.

To avoid poor prognosis in advanced urothelial carcinoma, systemic chemotherapy has been established and M-VAC therapy (methotrexate, vinblastine, doxorubicin and cisplatin), was proposed by the Memorial Sloan Kettering Cancer Center in 1985, which has been widely accepted as a first line regimen of chemotherapy (14). However, cardiotoxicity from doxorubicin is still a risk, and may be in doubt when using this therapeutic protocol. Additionally, significant adverse drug reactions, including bone marrow suppression and stomatitis has been observed, and despite a high response rate is, the survival rate seems still poor (15). Regimens with MEC (methotrexate, epirubicin and cisplatin) have been introduced since 1996 in urothelial carcinoma in the bladder, with wide acceptance in Asian patient groups 
(16) and have been established as efficacy as M-VAC (17). Thereafter, regimens with MEC have been used in our clinical institute.

Gemcitabine plus cisplatin (GC) has also been used and provides similar survival rate benefits to M-VAC, with a better safety profile and tolerability, whether for adjuvant therapy or neoadjuvant therapy (18). With fewer instances of neutropenic fever, mucositis, body weight loss and fatigue, it offered better compliance and acceptance in patients receiving GC than M-VAC did (19).

In our clinical Institute, each of the two regimens, MEC and GC, have been widely used in patients with advanced urothelial carcinoma in the upper and lower urinary tracts after radical surgery, as adjuvant chemotherapy. To our knowledge, there is no direct comparison of these two regimens available for advanced UTUCs. Thus, we try to identify patients with advanced UTUCs, after a definite radical nephroureterectomy and bladder cuff excision could benefit from these two regimens.

\section{Patients and Methods}

From January 2002 to December 2007, we retrospectively evaluated 279 patients with UTUC to receive radical nephroureterectomy and bladder cuff excision with lymph node dissection (open or laparoscopic method) at Taichung Veterans General Hospital. Patients with incomplete data, localized disease ( $\leq \mathrm{pT} 2 \mathrm{~N} 0 \mathrm{M} 0)$, metastasis disease at diagnosis, non-urothelial carcinoma and any who had received neoadjuvant therapy were excluded. Preoperative contrast-enhanced computed tomography was routinely performed and discussion with radiologist specific in the urinary tract. Pathological specimens were evaluated by a pathologist with expertise in the urinary tract in our institute, and the staging was recorded base on TNM classification.

Follow-up protocol included contrast compute tomography, urine cytology, a cystourethroscope and chest plain film. Patients were evaluated every 3 months for the first two years, every 6 months for the next two years and annually after. Inadequate evaluation and follow up of less than two years was excluded based on this protocol.

A total 70 patients were recorded. Adjuvant chemotherapy had been started within 3 months after definite radical surgery. The standard MEC consisted of methotrexate $\left(30 \mathrm{mg} / \mathrm{m}^{2}\right)$, epirubicin (30 $\left.\mathrm{mg} / \mathrm{m}^{2}\right)$ and cisplatin $\left(70 \mathrm{mg} / \mathrm{m}^{2}\right)$ administered every 3 weeks, for 4 6 courses in a cycle. Methotrexate was administered on day 1, while epirubicin and cisplatin were administered on day 2 . If after $24 \mathrm{~h}$ the CCR (Creatinine Clearance Rate) was $<60 \mathrm{cc} / \mathrm{min}$, cisplatin would be divided into two $35 \mathrm{mg} / \mathrm{m}^{2}$ courses on day 2 and day 3 . The protocol of GC consists of being administered every 3 weeks, in a 4-6 courses cycle, using a combination of gemcitabine and cisplatin with gemcitabine $1,200 \mathrm{mg} / \mathrm{m}^{2}$ on days 1 and 8 , plus cisplatin 70 $\mathrm{mg} / \mathrm{m}^{2}$ intravenously on day 9 . If after $24 \mathrm{~h} \mathrm{CCR}$ was $<60 \mathrm{cc} / \mathrm{min}$, cisplatin would be divided into two $35 \mathrm{mg} / \mathrm{m}^{2}$ courses on day 9 and 10. If it was needed, the next course would be delayed until after recovery of the absolute neutrophil count $\leq 500 / \mathrm{mm}^{3}$ and the platelet count $\leq 100,000 / \mathrm{mm}^{3}$ when G-CSF would be administered for neutropenia. This study has the institute with human ethical issue and the institute review board number was CE13240A-3.
Clinical characteristics were compared using both the Student's t-test and Pearson's Chi-square risk analysis. The definition of recurrence includes bladder recurrence, local recurrence or recurrence at the contralateral kidney, while disease-free survival (DFS) was also recorded. Cancer- specific survival (CSS) was defined as disease specific death, and overall Survival (OS) was defined as all cause death. Kaplan-Meier survival curve estimates and log-rank tests were used to determine the association of DFS, CSS and OS between these two groups of regimens and significance would be accepted at $p<0.05$.

Univariate and multivariate analysis using the Cox proportional hazards model were used to predict the DFS, CSS and OS. The variables with $p$-values $<0.2$ in the univariate analysis were checked into the multivariate model where statistical significance would be accepted at $p<0.05$ in the multivariate model. All statistical tests were carried out using IBM SPSS version 22.

\section{Results}

Table I summarizes the characteristics in a total of 70 patients among the two patient groups, MEC group $(n=30)$ and GC group $(n=40)$. The mean age and BMI (Body Mass Index) appear to possess no statistical differences among the two groups. Risk factors such as smoking, history of uremia, having received a transplant, pre-operative creatinine and pre-operative creatinine $>1.5$ also showed no significant differences between the two groups. In consideration of the pathology stage, there were $0 / 8 / 17 / 5$ patients of pT1/T2/T3/T4 in the MEC group, and 3/5/25/7 patients of $\mathrm{pT} 1 / \mathrm{T} 2 / \mathrm{T} 3 / \mathrm{T} 4$ respectively in the GC group $(p=0.240)$. Additionally, there were $24 / 3 / 3 / 0$ patients of $\mathrm{pN} 0 / \mathrm{N} 1 / \mathrm{N} 2 / \mathrm{N} 3$ in the MEC group, and 29/4/6/1 patients of pN0/N1/N2/N3 respectively in the GC group $(p=0.750)$. In consideration of tumor grading, there were $7 / 23$ patients of Grade $2 / 3$ in the MEC group, and 5/35 patients of Grade $2 / 3$ respectively in the GC group $(p=0.234)$. There was also no significance in multiple lesions, angiolymphatic invasion, surgical margin, positive lymph node and total lymph node number among the two groups. The only significant difference between the two groups may be found in the follow up time, which was relatively longer in the MEC group (45.91 \pm 29.61 months $v s$. $29.35 \pm 13.41$ months, $p=0.003$ ).

The Kaplan-Meier survival curve was used in the evaluation of DFS, CSS and OS among the two groups, which showed no statistical significance among the MEC group and GC group. Figure 1a shows that there is no significance among the two groups in DFS, where the median time in MEC vs. GC was $16.09 \pm 8.252$ $(95 \% \mathrm{CI}=0.000-32.272)$ and $20.66 \pm 7.39(95 \% \mathrm{CI}=6.187-$ 35.144 ). Figure $1 \mathrm{~b}$ and $\mathrm{c}$ shows that there was no significant difference in CSS and OS among the two regimens, and both did not reach the medial survival time. The 2-year DFS, CSS and OS rate among MEC vs. GC were $63.3 \%$ vs. 64.7\%, $81.6 \%$ vs. $87.3 \%$ and $75.5 \%$ vs. $85.0 \%$, respectively. Additionally, the 5-year DFS, CSS and OS rate among MEC 
Table I. Clinical and pathology characteristics of cohorts $(N=70)$.

\begin{tabular}{|c|c|c|c|c|c|}
\hline \multirow{2}{*}{$\begin{array}{l}\text { Category } \\
\text { Gender }\end{array}$} & \multicolumn{2}{|c|}{$\operatorname{MEC}(\mathrm{N}=30)$} & \multicolumn{2}{|c|}{$\mathrm{GC}(\mathrm{N}=40)$} & \multirow[t]{2}{*}{$p$-Value } \\
\hline & & & & & \\
\hline Male & 19 & $63.30 \%$ & 21 & $52.50 \%$ & 0.365 \\
\hline Female & 11 & $36.70 \%$ & 19 & $47.50 \%$ & \\
\hline \multicolumn{6}{|l|}{ Characteristics } \\
\hline Age & 60.03 & \pm 11.56 & 62.73 & \pm 12.04 & 0.422 \\
\hline Body height & 160.86 & \pm 7.19 & 160.67 & \pm 8.18 & 0.921 \\
\hline Body weight & 64.54 & \pm 7.24 & 62.14 & \pm 10.22 & 0.276 \\
\hline Body mass index & 24.99 & \pm 2.82 & 24.04 & \pm 3.35 & 0.213 \\
\hline \multicolumn{6}{|l|}{ Risk } \\
\hline Smoke & 13 & $43.30 \%$ & 11 & $27.50 \%$ & 0.167 \\
\hline Transplant & 1 & $3.30 \%$ & 5 & $12.50 \%$ & 0.175 \\
\hline Pre op Creat & 1.36 & \pm 0.38 & 1.43 & \pm 0.76 & 0.642 \\
\hline Crea $>1.5$ & 12 & $40.00 \%$ & 17 & $42.50 \%$ & 0.834 \\
\hline \multicolumn{6}{|l|}{ Pathology } \\
\hline \multicolumn{6}{|l|}{ pT } \\
\hline $\mathrm{T} 1$ & 0 & $0.00 \%$ & 3 & $7.50 \%$ & 0.24 \\
\hline $\mathrm{T} 2$ & 8 & $26.70 \%$ & 5 & $12.50 \%$ & \\
\hline $\mathrm{T} 3$ & 17 & $56.70 \%$ & 25 & $62.50 \%$ & \\
\hline $\mathrm{T} 4$ & 5 & $16.70 \%$ & 7 & $17.50 \%$ & \\
\hline \multicolumn{6}{|l|}{$\mathrm{pN}$} \\
\hline No & 24 & $80.00 \%$ & 29 & $72.50 \%$ & 0.75 \\
\hline N1 & 3 & $10.00 \%$ & 4 & $10.00 \%$ & \\
\hline $\mathrm{N} 2$ & 3 & $10.00 \%$ & 6 & $15.00 \%$ & \\
\hline $\mathrm{N} 3$ & 0 & $0.00 \%$ & 1 & $2.50 \%$ & \\
\hline \multicolumn{6}{|l|}{ Tumor Gr } \\
\hline G2 & 7 & $23.30 \%$ & 5 & $12.50 \%$ & 0.234 \\
\hline G3 & 23 & $76.70 \%$ & 35 & $87.50 \%$ & \\
\hline CIS & 4 & $13.30 \%$ & 3 & $7.50 \%$ & 0.421 \\
\hline Multiple & 11 & $36.70 \%$ & 19 & $47.50 \%$ & 0.365 \\
\hline Angiolymphatic invasion & 12 & $40.00 \%$ & 18 & $45.00 \%$ & 0.676 \\
\hline Surgical margin & 6 & $20.00 \%$ & 4 & $10.00 \%$ & 0.237 \\
\hline \multicolumn{6}{|l|}{ Node } \\
\hline Node positive Number & 1.27 & \pm 5.5 & 0.95 & \pm 2.35 & 0.745 \\
\hline Node total Number & 2.57 & \pm 5.81 & 1.6 & \pm 2.97 & 0.367 \\
\hline \multicolumn{6}{|l|}{ Location } \\
\hline Right & 12 & $40.00 \%$ & 21 & $52.50 \%$ & 0.336 \\
\hline Left & 17 & $56.70 \%$ & 19 & $47.50 \%$ & \\
\hline Bilateral & 1 & $3.30 \%$ & 0 & $0.00 \%$ & \\
\hline Calyx & 6 & $20.00 \%$ & 15 & $37.50 \%$ & 0.114 \\
\hline Renal plevis & 20 & $66.70 \%$ & 29 & $72.50 \%$ & 0.598 \\
\hline Ureter $\mathrm{U} / 3$ & 8 & $26.70 \%$ & 7 & $17.50 \%$ & 0.355 \\
\hline Ureter $\mathrm{M} / 3$ & 6 & $20.00 \%$ & 11 & $27.50 \%$ & 0.469 \\
\hline Ureter $\mathrm{L} / 3$ & 7 & $23.30 \%$ & 9 & $22.50 \%$ & 0.935 \\
\hline \multicolumn{6}{|l|}{ Follow up } \\
\hline Follow up time (M) & 45.91 & \pm 29.61 & 29.35 & \pm 13.41 & 0.003 \\
\hline
\end{tabular}

Continous variable analysis use Student's $t$-test, mean \pm SD. Categorical variables analysis use Pearson Chi-Square test, n (\%)

vs. GC were $39.4 \%$ vs. $44.4 \%, 81.6 \%$ vs. $74.7 \%$ and $70.8 \%$ vs. $52.1 \%$, respectively. The efficacy of MEC and GC in two independent risk factors, lymph node positive and creatinine, was discussed separately.

The patients with positive lymph nodes appeared to have poor prognosis, while the 2-year and 5-year CSS among our population was $55.6 \%$ vs. $93.7 \%$ and $47.6 \%$ vs. $87.5 \%$ $(p=0.000)$. The efficacy among this subgroup was examined and Figure $2 \mathrm{a}, \mathrm{b}$ and $\mathrm{c}$ reveals that there is no significant difference in DFS, CSS and OS rates among MEC and GC in patients with positive lymph nodes $(n=16,6$ in MEC group and 10 in GC group). The median time of DFS in MEC and GC in lymph node positive patients was $5.13 \pm 2.23 \quad(95 \% \mathrm{CI}=0.748-9.502) \quad$ and $10.09 \pm 3.97$ 

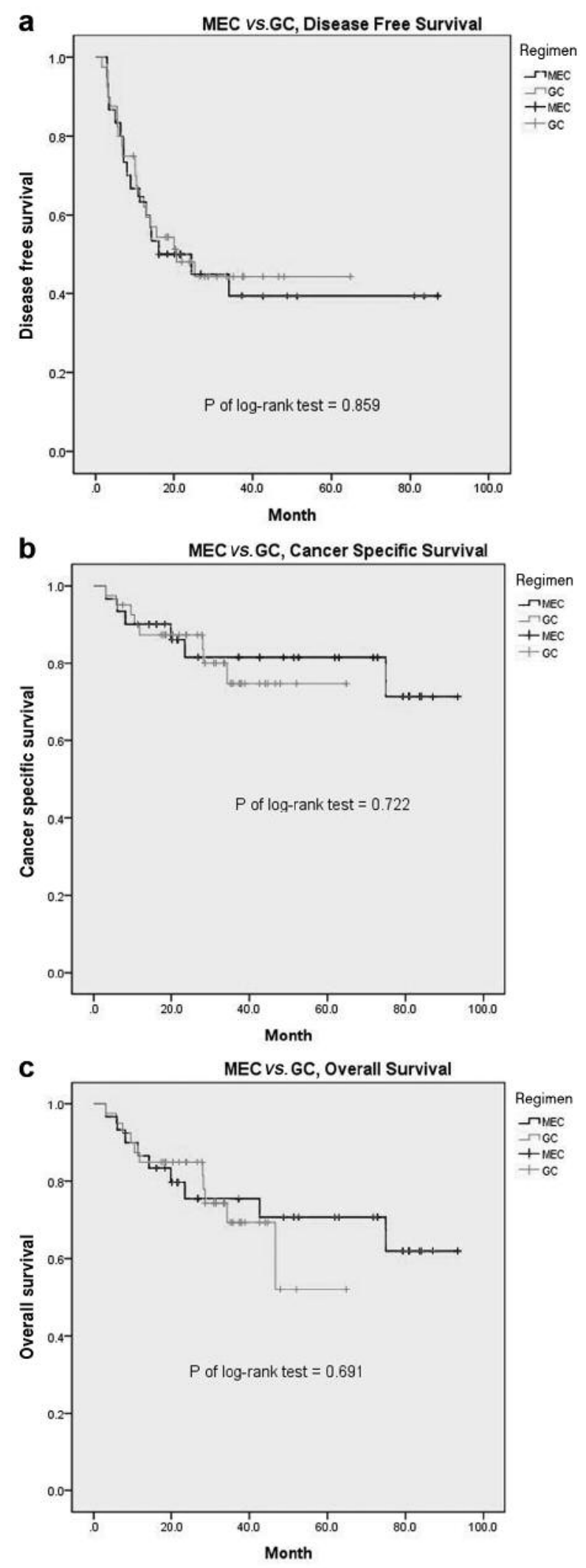

Figure 1. Kaplan-Meier survival curve evaluation of DFS (a), CSS (b) and $O S(c)$ in patients with UTUC who received MEC $(n=30)$ and $G C(n=40)$.
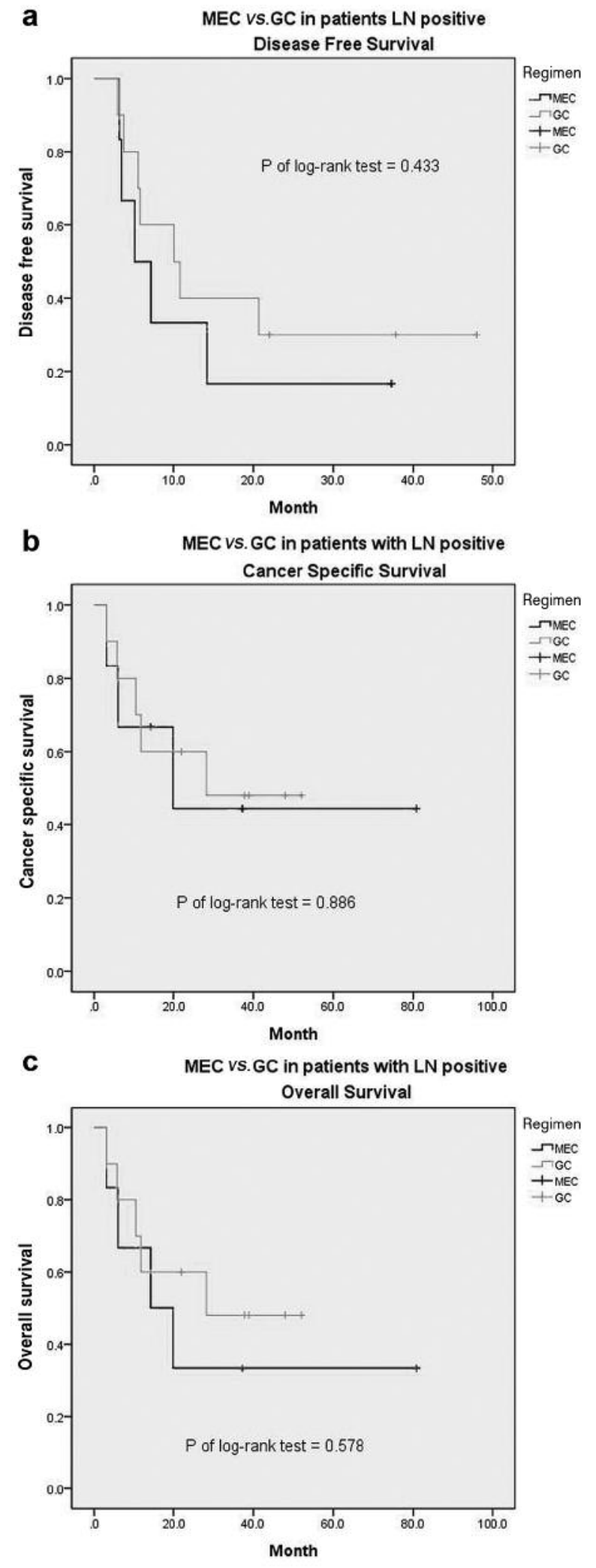

Figure 2. Kaplan-Meier survival curve evaluation for DFS (a), CSS (b) and $O S(c)$ in patients with lymph node positive UTUC $(N=16)$ who received $\operatorname{MEC}(n=6)$ and $G C(n=10)$. 
(95\% CI $=2.297-17.875$ ). Both CSS and OS rates among the two regimens in this subgroup did not reach the median survival time.

Patients with preoperative creatinine $>1.5$ also appeared to have a poor prognosis, and the 2-year and 5-year OS among our population was $71.9 \%$ vs. $87.4 \%$ and $45.2 \%$ vs. $83.9 \%$ ( $p=0.013$ ). The efficacy among this subgroup was also examined. Figure $3 \mathrm{a}, \mathrm{b}$ and $\mathrm{c}$ reveals that there is no significant difference in DFS, CSS and OS rates among MEC and GC in patients with creatinine $>1.5(n=29,12$ in MEC group and 17 in GC group). All DFS, CSS and OS in patients who received MEC and GC in this subgroup did not reach the median survival time. Univariate and multivariate analysis with the Cox proportional hazards model were used to predict the risk factors in DFS, CSS and OS.

In Table II, the model of DFS shows that performance state $(\mathrm{HR}=2.506,95 \% \mathrm{CI}=1.034-6.071)$ and lymph node positive $(\mathrm{HR}=2.520,95 \% \mathrm{CI}=1.108-5.734)$ were two independent risk factors for disease recurrence. BMI, uremia, multiple lesions, angiolymphatic invasion, renal calyx tumor, renal pelvis tumor and bladder tumor at diagnosis showed no statistical significance in a multivariate analysis of the DFS model.

In Table III, the model for CSS is discussed. Age $(\mathrm{HR}=1.097,95 \% \mathrm{CI}=1.025-1.175)$, gender (female $v s$. male, $\mathrm{HR}=0.012,95 \% \mathrm{CI}=0.000-0.966)$ and positive lymph node $(\mathrm{HR}=40.628,95 \% \mathrm{CI}=6.283-262.719)$ were the three independent risk factors for cancer-specific mortality. BMI, performance state, multiple lesions, CIS, Creatinine $>1.5$ and renal calyx tumor showed no statistical significance in a multivariate analysis of the CSS model.

Table IV reveals univariate analysis and multivariate analysis in the OS model. When compared to CSS, age $(\mathrm{HR}=1.074,95 \% \mathrm{CI}=1.023-1.128)$ was the same significant risk factor. There are three major differences between the OS and CSS models: gender was not related to any risk for all causes of death in this group, but BMI (HR=0.793, 95\% $\mathrm{CI}=0.658-0.957) \quad$ and creatinine $>1.5 \quad(\mathrm{HR}=10.214$, $95 \% \mathrm{CI}=3.237-32.228)$, which were not a statistical significance in the CSS model, displayed a significance in the OS model. Among the three univariate and multivariate models, a regimen of adjuvant chemotherapy, MEC or GC, was not a risk factor towards DFS, CSS and OS.

In summary, there is no significant difference in MEC and GC in all UTUC patients, UTUC patients who were lymph node positive or UTUC patients with creatinine $>1.5$. Performance state and positive lymph nodes were independent risk factors for cancer recurrence (including bladder recurrence). Age, gender and positive lymph node were independent risk factors for cancer specific mortality. Age, BMI, positive lymph node and creatinine $>1.5$ were independent risk factors for all causes of mortality.
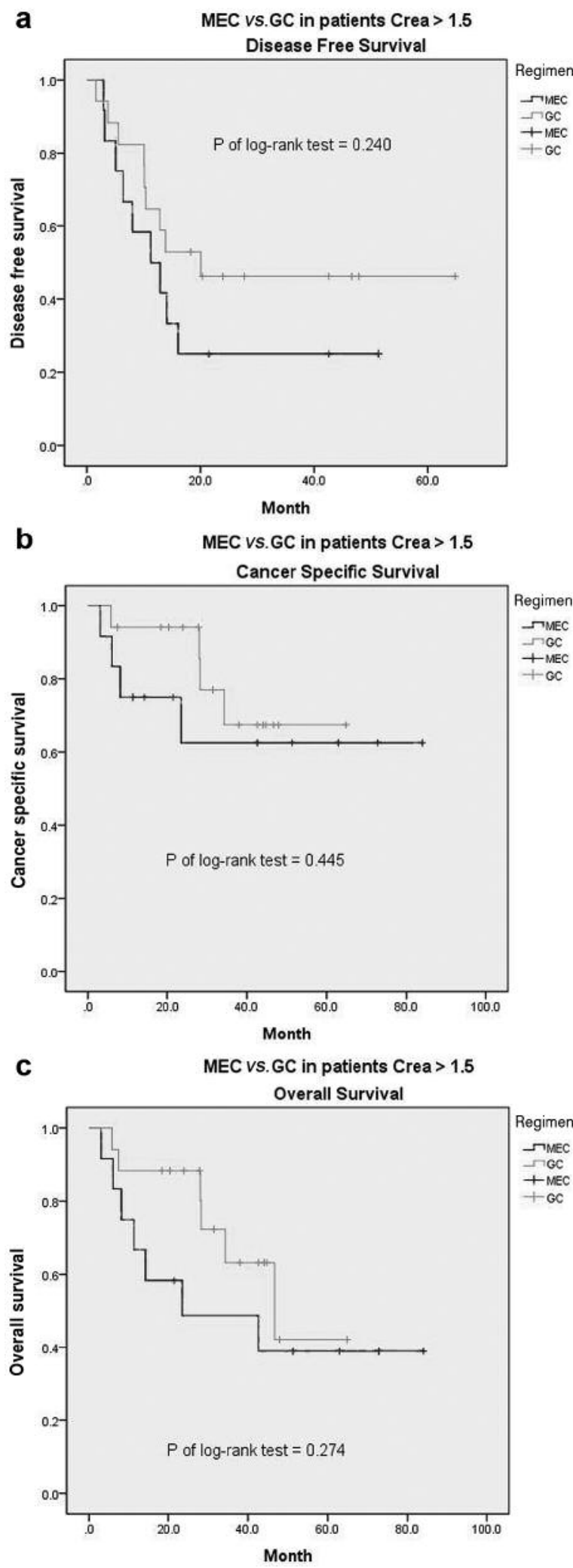

Figure 3. Kaplan-Meier survival curve evaluation for DFS (a), CSS (b) and $O S(c)$ in patients with UTUC and preoperative creatinine $>1.5$ $(N=29)$ who received $M E C(n=12)$ and $G C(n=17)$. 
Table II. Univariate and multivariate Cox proportional hazard regression analysis for prediction of disease-free survival.

\begin{tabular}{|c|c|c|c|c|}
\hline \multirow[b]{2}{*}{ Category } & \multicolumn{2}{|c|}{ Univariate } & \multicolumn{2}{|c|}{ Multivariate } \\
\hline & $\mathrm{HR}(95 \% \mathrm{CI})$ & $p$-Value & $\mathrm{HR}(95 \% \mathrm{CI})$ & $p$-Value \\
\hline Age & $1.023(0.541-1.935)$ & 0.944 & & \\
\hline Gender (Female $v s$. male) & $0.701(0.362-1.357)$ & 0.292 & & \\
\hline BMI & $0.931(0.841-1.031)$ & 0.17 & $0.951(0.855-1.057)$ & 0.352 \\
\hline Performance state (ECOG 2 vs. 1) & $2.289(1.044-5.020)$ & 0.039 & $2.506(1.034-6.071)$ & $0.042 *$ \\
\hline Uremia & $1.863(0.726-4.780)$ & 0.195 & $1.250(0.394-3.963)$ & 0.704 \\
\hline Smoking & $1.066(0.545-2.088)$ & 0.851 & & \\
\hline Multiple Lesion & $2.363(1.236-4.516)$ & 0.009 & $1.610(0.665-3.896)$ & 0.291 \\
\hline CIS & $1.500(0.585-3.848)$ & 0.399 & & \\
\hline Grade (Gr $3 v s$. Gr 2) & $1.231(0.514-2.945)$ & 0.641 & & \\
\hline $\mathrm{N}$ positive & $2.308(1.155-4.614)$ & 0.018 & $2.520(1.108-5.734)$ & $0.028^{*}$ \\
\hline Angiolymphatic invasion & $1.664(0.879-3.150)$ & 0.118 & $1.513(0.739-3.099)$ & 0.258 \\
\hline Surgical Margin & $1.078(0.420-2.770)$ & 0.875 & & \\
\hline $\mathrm{Cr}>1.5$ & $1.403(0.741-2.657)$ & 0.298 & & \\
\hline Calyx & $2.102(1.093-4.043)$ & 0.026 & $1.698(0.743-3.880)$ & 0.209 \\
\hline Renal pelvis & $1.828(0.837-3.991)$ & 0.13 & $1.295(0.537-3.126)$ & 0.565 \\
\hline $\mathrm{U} / 3$ ureter & $1.423(0.673-3.009)$ & 0.356 & & \\
\hline $\mathrm{M} / 3$ ureter & $1.264(0.613-2.607)$ & 0.525 & & \\
\hline $\mathrm{L} / 3$ ureter & $1.132(0.534-2.398)$ & 0.747 & & \\
\hline Urinary bladder lesion & $2.399(0.728-7.901)$ & 0.15 & $3.535(0.747-16.728)$ & 0.111 \\
\hline Adjuvant chemotherapy (MEC $v s$. GC) & $0.944(0.498-1.789)$ & 0.859 & & \\
\hline
\end{tabular}

HR, Harzard ratio; CI, confidence interval; BMI, body mass index; CIS, carcinoma in situ.

\section{Discussion}

Although UTUC is morphologically similar to bladder cancer, there are occasional phenotypic and genotypic (genetic and epigenetic) differences between UTUC and bladder cancer (11). Thus, despite having the same origin as urothelial carcinoma, further discussion regarding UTUCs is still needed. Adjuvant chemotherapy with a cisplatin base regimen was first introduced in 1985 and revealed a $21 \%$ partial remission rate in urothelial carcinoma patients (14) and had been widely accepted until now. Many of publications have reported on the benefits of adjuvant chemotherapy in urothelial carcinoma of the bladder, where one meta-analysis declared a $25 \%$ relative reduction in the risk of death for chemotherapy (20). Although there is still controversy, many of the studies have established that there are survival benefits with adjuvant chemotherapy in UTUCs $(21,22)$.

Kwak et al. directly compared 32 patients with UTUCs who were receiving adjuvant chemotherapy (M-VAC and GC), with 11 patients receiving only a surgical resection, revealing the benefits in DFS (include bladder recurrence) and OS (21). Suzuki et al. further reported DFS and OS in advanced UTUCs, and even further in patients with lymph node involvement (22).
In our Institute, the regimens mentioned above (MEC and GC) have been widely used up until now for advanced lower urinary tract and upper urinary tract urothelial carcinoma with tolerable side effects. Despite M-VAC having been widely used around the world, the side effects of cardiotoxicity and other symptoms make it an undesirable option. MEC has been reported to have a similar efficacy as M-VAC, but with a less toxic effect $(17,23)$, a factor which was more accepted in our institute. Moreover, the efficacy of GC has been proved in urothelial cancer (19). To our knowledge, there has been no direct comparison of these two regimens, MEC and GC. Which regimen may prove to be superior may be what we need to consider as we discuss more on this topic in the future.

During our investigation, the efficacy of both MEC and GC was comparable when considering DFS, and because bladder recurrence was included, this may reflect the efficacy of systemic chemotherapy. Additionally, there is no difference in CSS and OS, which further suggests that MEC is an effective protocol for UTUCs.

Lymph node-positive patients were discussed separately because their condition has been reported in many publications as an independent risk factor in UTUCs. Kwak et al. state that positive lymph nodes possess a significant risk of recurrence and for all causes of death (21). Kazutoshi 
Table III. Univariate and multivariate Cox proportional hazard regression analysis for prediction of cancer-specific survival.

\begin{tabular}{|c|c|c|c|c|}
\hline \multirow[b]{2}{*}{ Category } & \multicolumn{2}{|c|}{ Univariate } & \multicolumn{2}{|c|}{ Multivariate } \\
\hline & HR $(95 \%$ CI $)$ & $p$-Value & $\mathrm{HR}(95 \% \mathrm{CI})$ & $p$-Value \\
\hline Age & $1.049(0.996-1.105)$ & 0.071 & $1.097(1.025-1.175)$ & $0.008^{*}$ \\
\hline Gender (Female vs. male) & $0.106(0.014-0.815)$ & 0.031 & $0.012(0.000-0.966)$ & $0.048^{*}$ \\
\hline BMI & $0.862(0.709-1.048)$ & 0.137 & $0.937(0.696-1.262)$ & 0.67 \\
\hline Performance state (ECOG 2 vs. 1) & $2.690(0.833-8.686)$ & 0.098 & $6.939(0.791-60.915)$ & 0.08 \\
\hline Uremia & $0.042(0.000-91.475)$ & 0.418 & & \\
\hline Smoking & $0.965(0.322-2.892)$ & 0.95 & & \\
\hline Multiple Lesion & $3.853(1.198-12.396)$ & 0.024 & $2.675(0.260-27.508)$ & 0.408 \\
\hline CIS & $2.892(0.795-10.528)$ & 0.107 & $5.114(0.595-43.983)$ & 0.137 \\
\hline Grade (Gr 3 vs. Gr 2) & $1.298(0.290-5.808)$ & 0.733 & & \\
\hline $\mathrm{N}$ positive & $5.921(2.045-17.143)$ & 0.001 & $40.628(6.283-262.719)$ & $0.001^{*}$ \\
\hline Angiolymphatic invasion & $1.853(0.641-5.351)$ & 0.255 & & \\
\hline Surgical Margin & $1.066(0.238-4.769)$ & 0.933 & & \\
\hline $\mathrm{Cr}>1.5$ & $2.268(0.772-6.660)$ & 0.136 & $1.867(0.476-7.316)$ & 0.37 \\
\hline Calyx & $5.169(1.711-15.614)$ & 0.004 & $1.449(0.158-13.285)$ & 0.743 \\
\hline Renal pelvis & $2.597(0.581-11.621)$ & 0.212 & & \\
\hline $\mathrm{U} / 3$ ureter & $1.341(0.413-4.356)$ & 0.626 & & \\
\hline $\mathrm{M} / 3$ ureter & $1.277(0.398-4.099)$ & 0.681 & & \\
\hline $\mathrm{L} / 3$ ureter & $0.556(0.124-2.493)$ & 0.443 & & \\
\hline Urinary bladder lesion & $0.045(0.000-1644.968)$ & 0.564 & & \\
\hline Adjuvant chemotherapy (MEC vs. GC) & $1.224(0.400-3.750)$ & 0.723 & & \\
\hline
\end{tabular}

HR, Harzard ratio; CI, confidence interval; BMI, body mass index; CIS, carcinoma in situ.

Table IV. Univariate and multivariate Cox proportional hazard regression analysis for prediction of overall survival.

\begin{tabular}{|c|c|c|c|c|}
\hline \multirow[b]{2}{*}{ Category } & \multicolumn{2}{|c|}{ Univariate } & \multicolumn{2}{|c|}{ Multivariate } \\
\hline & HR $(95 \%$ CI $)$ & $p$-Value & HR $(95 \% \mathrm{CI})$ & $p$-Value \\
\hline Age & $1.040(0.997-1.084)$ & 0.068 & $1.074(1.023-1.128)$ & $0.004 *$ \\
\hline Gender (Female vs. male) & $0.354(0.117-1.067)$ & 0.065 & $0.414(0.096-1.784)$ & 0.237 \\
\hline BMI & $0.821(0.693-0.973)$ & 0.023 & $0.793(0.658-0.957)$ & $0.016^{*}$ \\
\hline Performance state (ECOG 2 vs. 1) & $2.344(0.844-6.505)$ & 0.102 & $4.684(1.207-18.180)$ & $0.026^{*}$ \\
\hline Uremia & $0.479(0.064-3.596)$ & 0.474 & & \\
\hline Smoking & $0.922(0.367-2.318)$ & 0.864 & & \\
\hline Multiple Lesion & $2.361(0.959-5.815)$ & 0.062 & $1.057(0.320-3.497)$ & 0.927 \\
\hline CIS & $1.915(0.556-6.596)$ & 0.303 & & \\
\hline Grade (Gr 3 vs. Gr 2) & $0.677(0.246-1.869)$ & 0.452 & & \\
\hline $\mathrm{N}$ positive & $3.791(1.565-9.183)$ & 0.003 & $10.214(3.237-32.228)$ & $0.001 *$ \\
\hline Angiolymphatic invasion & $1.425(0.592-3.431)$ & 0.429 & & \\
\hline Surgical Margin & $1.095(0.320-3.739)$ & 0.885 & & \\
\hline $\mathrm{Cr}>1.5$ & $3.093(1.217-7.860)$ & 0.018 & $3.178(1.029-9.819)$ & $0.045^{*}$ \\
\hline Calyx & $3.667(1.504-8.938)$ & 0.004 & $3.623(0.996-13.175)$ & 0.051 \\
\hline Renal pelvis & $1.782(0.595-5.228)$ & 0.302 & & \\
\hline $\mathrm{U} / 3$ ureter & $1.116(0.401-3.109)$ & 0.833 & & \\
\hline $\mathrm{M} / 3$ ureter & $0.842(0.279-2.539)$ & 0.76 & & \\
\hline $\mathrm{L} / 3$ ureter & $0.575(0.168-1.965)$ & 0.377 & & \\
\hline Urinary bladder lesion & $0.044(0.000-171.795)$ & 0.46 & & \\
\hline Adjuvant chemotherapy (MEC vs. GC) & $1.207(0.476-3.062)$ & 0.691 & & \\
\hline
\end{tabular}

HR, Harzard ratio; CI, confidence interval; BMI, body mass index; CIS, carcinoma in situ . 
et al. further discuss the effect of adjuvant chemotherapy in patients with lymph node positive UTUCs as having a $48 \%$ reduction in the risk of recurrence, and a $64 \%$ reduction in the risk of cancer specific mortality (24). In our investigation, lymph node positive appears to be a poor prognosis, while having a higher risk of recurrence (HR $2.520,95 \% \mathrm{CI}=1.108-5.5734)$, cancer specific mortality (HR $40.628,95 \% \mathrm{CI}=6.283-262.719)$ and for all causes of death (HR 10.214, 95\%CI=3.237-32.228). MEC and GC appear to display a comparable efficacy with regards to DFS, CSS and OS in this subgroup of patients.

A level of creatinine $>1.5$ prior to surgery reflects relatively poor renal function and in our experience, may be an additional risk of uremia for patients who are a candidate for a radical nephrectomy. This condition would also indicate whether or not patients could tolerate a complete course of chemotherapy. This is a major difference from urothelial carcinoma in the upper tract and urinary bladder, which could preserve the bilateral kidney with relatively better renal function. Shuichi et al. declare preoperative creatinine as an independent risk factor in regards to CSS in patients with UTUCs (25). Our results show that despite there being no significance in CSS $(p=0.126)$, the prognosis is significantly poorer in OS (5-year survival $45.2 \%, p=0.013$ ). This may explain the deterioration of renal function after a radical nephrectomy and how cisplatin based chemotherapy would be a risk that can't be ignored and may introduce to all cause death. Respectively, MEC and GC were examined in this subgroup and displayed no differences for all causes of death, which may indicate the comparable efficacy and toxicity.

As outlined in most publications, age and performance state with ECOG may reflect the patient's disabilities and the disease severity. Colin et al. further describe that age, ECOG status and a surgical delay of more than 3 months would cause a risk in prognosis (26). When compared to our result, the higher ECOG score also indicates a higher risk of recurrence $(\mathrm{HR}=2.506,95 \% \mathrm{CI}=1.034-6.071, p=0.042)$ and for all causes of death $(\mathrm{HR}=4.684,95 \% \mathrm{CI}=1.207-18.180$, $p=0.026)$. It appears likely that age seems to be an independent risk for cancer specific death $(\mathrm{HR}=1.097$, $95 \% \mathrm{CI}=1.025-1.175, p=0.008)$ and for all causes of death $(\mathrm{HR}=1.074,95 \% \mathrm{CI}=1.023-1.128, p=0.004)$.

$\mathrm{BMI}$ is thought to be another risk factor for poor prognosis, which may reflect the cachexia condition of the patient. Liu et al. state that being preoperatively underweight is an independent predictor of unfavorable recurrence-free survival, along with cancer-specific survival in Chinese patients with UTUC who were treated with a radical nephroureterectomy (27). That outcome was the same in comparison with our result, with regards to OS (HR 0.793, 95\% CI 0.658-0.957, $p=0.016$ ).

Although uremia is an established risk factor for urothelial carcinoma, there was no significance in our cohort. This may due to cisplatin based regimens which should be used in concern of toxicity and side effects in this group of patients and this may lead to a selection bias. Uremic patients with UTUCs under hemodialysis would be excluded in and all of the uremia patients in our population in this cohort had received a renal transplant prior, which may be the reason for the deviation.

In view of its retrospective nature, this study may be limited by its small sample size and short follow up time. By using a larger sample, future prospective studies may give us better answers towards the efficacy of these two regimens in UTUCs. However, due to the current lack of study focus on MEC and GC in UTUCs, there may still be some value in our study.

\section{Conclusion}

In patients with upper tract urothelial carcinoma, MEC has a non-inferior efficacy to GC when considering cancer recurrence, cancer specific survival and overall survival. Positive lymph nodes, preoperative creatinine $>1.5$, old age, a high ECOG state and low BMI are the independent risk factors for a poor prognosis in advanced upper tract urothelial carcinoma. Further multi-institutional, prospective studies are required to confirm these findings.

\section{Conflicts of Interest}

None of the contributing Authors have any conflict of interest, including specific financial interests or relationships and affiliations relevant to the subject matter or materials discussed in the manuscript.

\section{Ethical Approval}

This study has the institute with human ethical issue and the institute review board number was CE13240A-3.

\section{References}

1 Ferlay J, Shin HR, Bray F, Forman D, Mathers C and Parkin DM: Estimates of worldwide burden of cancer in 2008: GLOBOCAN 2008. Int J Cancer 127(12): 2893-2917, 2010.

2 Babjuk M, Burger M, Zigeuner R, Shariat SF, van Rhijn BWG, Compérat E, Sylvester RJ, Kaasinen E, Böhle A, Palou Redorta $\mathrm{J}$ and Rouprêt M; European Association of Urology: EAU guidelines on non-muscle-invasive urothelial carcinoma of the bladder: Update 2013. Eur Urol 64(4): 639-653, 2013.

3 Chen CH, Dickman KG, Moriya M, Zavadil J, Sidorenko VS, Edwards KL, Gnatenko DV, Wu L, Turesky RJ, Wu XR, Pu YS and Grollman AP: Aristolochic acid-associated urothelial cancer in Taiwan. Proc Natl Acad Sci USA 109(21): 82418246, 2012.

4 Cosentino M, Palou J, Gaya JM, Breda A, Rodriguez-Faba O and Villavicencio-Mavrich H: Upper urinary tract urothelial cell carcinoma: Location as a predictive factor for concomitant bladder carcinoma. World J Urol 31(1): 141-145, 2013. 
5 Seisen T, Granger B, Colin P, Léon P, Utard G, Renard-Penna R, Compérat E, Mozer P, Cussenot O, Shariat SF and Rouprêt M: A Systematic Review and Meta-analysis of Clinicopathologic Factors Linked to Intravesical Recurrence after Radical Nephroureterectomy to Treat Upper Tract Urothelial Carcinoma. Eur Urol 67(6): 1122-1133, 2015

6 Li WM, Shen JT, Li CC, Ke HL, Wei YC, Wu WJ, Chou YH and Hung $\mathrm{CH}$ : Oncologic outcomes following three different approaches to the distal ureter and bladder cuff in nephroureterectomy for primary upper urinary tract urothelial carcinoma. Eur Urol 57(6): 963-969, 2010.

7 Margulis V, Shariat SF, Matin SF, Kamat AM, Zigeuner R, Kikuchi E, Lotan Y, Weizer A, Raman JD and Wood CG: The upper tract urothelial carcinoma collaboration. Outcomes of radical nephroureterectomy: A series from the upper tract urothelial carcinoma collaboration. Cancer 115(6): 1224-1233, 2009.

8 Abouassaly R, Alibhai SM, Shah N, Timilshina N, Fleshner N, Finelli A: Troubling outcomes from population-level analysis of surgery for upper tract urothelial carcinoma. Urology 76(4): 895$901,2010$.

9 Jeldres C, Sun M, Isbarn H, Lughezzani G, Budäus L, Alasker A, Shariat SF, Lattouf JB, Widmer H, Pharand D, Arjane P, Graefen M, Montorsi F, Perrotte P and Karakiewicz PI: A population-based assessment of perioperative mortality after nephroureterectomy for upper-tract urothelial carcinoma. Urology 75(2): 315-20, 2010.

10 Roupret M, Babjuk M, Compérat E, Zigeuner R, Sylvester R, Burger M, Cowan N, Böhle A, Van Rhijn BW, Kaasinen E, Palou $\mathrm{J}$ and Shariat SF; European Association of Urology: European Association of Urology. European guidelines on upper tract urothelial carcinomas: 2013 update. Eur Urol 63: 1059-1071, 2013.

11 Green DA, Rink M, Xylinas E, Matin SF, Stenzl A, Roupret M, Karakiewicz PI, Scherr DS and Shariat SF: Urothelial carcinoma of the bladder and the upper tract: Disparate twins. J Urol 189(4): 1214-1221, 2013.

12 Bellmunt J and Petrylak DP: New therapeutic challenges in advanced bladder cancer. Semin Oncol 39(5): 598-607, 2012.

13 van Oers JM, Zwarthoff EC, Rehman I, Azzouzi AR, Cussenot O, Meuth M, Hamdy FC and Catto JW: FGFR3 mutations indicate better survival in invasive upper urinary tract and bladder tumours. Eur Urol 55(3): 650-657, 2009.

14 Sternberg CN, Yagoda A, Scher HI, Watson RC, Ahmed T, Weiselberg LR, Geller N, Hollander PS, Herr HW and Sogani PC: Preliminary results of M-VAC (methotrexate, vinblastine, doxorubicin and cisplatin) for transitional cell carcinoma of the urothelium. J Urol 133: 403-407, 1985.

15 Sternberg CN, Yagoda A, Scher HI, Watson RC, Geller N, Herr HW, Morse MJ, Sogani PC, Vaughan ED and Bander N: M-VAC for advanced transitional cell carcinoma of the urothelium: Efficacy and patterns of response. Cancer 64: 2448-2458, 1989.

16 Cacciari N, Martoni A, Rossi AP, Turci P, Lelli G, Martinelli A, Maver P, Corrado F, Mannini D, Reggiani A, Concetti S, Martelli A, Melotti B, Cricca A, Zamagni C and Pannuti F: A new regimen of cisplatin, epirubicin and methotrexate (PEM-3) as primary chemotherapy for locally advanced bladder cancer. Tumori 82(4): 364-368, 1996.

17 Kuroda M, Kotake T, Akaza H, Hinotsu S and Kakizoe T: Efficacy of Dose-intensified MEC (Methotrexate, Epirubicin and Cisplatin) chemotherapy for advanced urothelial carcinoma: A prospective randomized trial comparing MEC and M-VAC
(Methotrexate, Vinblastine, Doxorubicin and Cisplatin). Jpn J Clin Oncol 28(8): 497-501, 1998.

18 Dash A, Pettus JA 4th, Herr HW, Bochner BH, Dalbagni G, Donat SM, Russo P, Boyle MG, Milowsky MI and Bajorin DF: A role for neoadjuvant gemcitabine plus cisplatin in muscleinvasive urothelial carcinoma of the bladder: A retrospective experience. Cancer 113: 2471-2477, 2008.

19 Von der Maase H, Hansen SW, Roberts JT, Dogliotti L, Oliver T, Moore MJ, Bodrogi I, Albers P, Knuth A, Lippert CM, Kerbrat P, Sanchez Rovira P, Wersall P, Cleall SP, Roychowdhury DF, Tomlin I, Visseren-Grul CM and Conte PF: Gemcitabine and cisplatin versus methotrexate, vinblastine, doxorubicin, and cisplatin in advanced or metastatic bladder cancer: results of a large, randomized, multinational, multicenter, phase III study. J Clin Oncol 18: 3068-3077, 2000.

20 Vale CL: Adjuvant Chemotherapy in Invasive Bladder Cancer: A Systematic Review and Meta-Analysis of Individual Patient Data: Advanced Bladder Cancer (ABC) Meta-analysis Collaboration. Eur Urol 48(2): 189-199, 2005.

$21 \mathrm{Kwak} \mathrm{C}$, Lee SE, Jeong IG and Ku JH: Adjuvant systemic chemotherapy in the treatment of patients with invasive transitional cell carcinoma of the upper urinary tract. Urology 68: 53-57, 2006.

22 Suzuki S, Shinohara N, Harabayashi T, Sato S, Abe T and Koyanagi T: Impact of adjuvant systemic chemotherapy on postoperative survival in patients with high-risk urothelial cancer. Int J Urol 11: 456-460, 2004.

23 Matsui Y, Nishiyama H, Watanabe J, Teramukai S, Ono Y, Ohshima S, Fujimoto K, Hirao Y, Fukushima M and Ogqwa O: The current status of perioperative chemotherapy for invasive bladder cancer: A multi-institutional retrospective study in Japan. Int J Clin Oncol 10(2): 133-138, 2005.

24 Fujita K, Inamoto T, Yamamoto Y, Tanigawa G, Nakayama M, Mori N, Tsujihata M, Azuma H, Nonomura N and Uemura M: Role of adjuvant chemotherapy for lymph node-positive upper tract urothelial carcinoma and the prognostic significance of $\mathrm{C}$ reactive protein: A multi-institutional, retrospective study. IJU 22: 1006-1012, 2015.

25 Morizane S, Iwamoto H, Masago T, Yao A, Isoyama T, Sejima $\mathrm{T}$ and Takenaka A: Preoperative prognostic factors after radical nephroureterectomy in patients with upper urinary tract urothelial carcinoma. Int Urol Nephrol 45(1): 99-106, 2013.

26 Colin P, Irani J, Drouin SJ, Shariat SF and Rouprêt M: Prognostic factors of upper tract urothelial carcinomas and impact on survival: A systematic review for the yearly scientific report of the French National Association of Urology. Prog Urol 24(15): 1000-1010, 2014.

27 Liu JY, Li YH, Liu ZW, Zhang ZL, Ye YL, Yao K, Jiang LJ, Han $\mathrm{H}$, Qin ZK and Zhou FJ: Influence of body mass index on oncological outcomes in patients with upper urinary tract urothelial carcinoma treated with radical nephroureterectomy. Int J Urol 21(2): 136-142, 2014. 